\title{
Exposing relative endoscopy in unitary symmetric spaces
}

\author{
Jason KC Polák
}

Correspondence: jpolak@jpolak.org Department of Mathematics, McGil University, RM1005, 805 Sherbrooke St West, H3A OB9 Montreal, Canada

\begin{abstract}
We introduce a new class of symmetric space orbital integrals important for applications in certain relative trace formula appearing in the theory of automorphic representations. We verify a fundamental lemma for $U_{2} \times U_{2} \hookrightarrow U_{4}$ via an explicit calculation, giving the first known example of endoscopy for symmetric spaces and showing strong evidence that there is a general theory of endoscopy lurking in this situation.
\end{abstract}

AMS subject classification: Primary 20G05

Keywords: Endoscopy; Orbital integral; Unitary group; Trace formula; Representation theory

\section{Introduction}

\section{Endoscopy and representations}

Endoscopy is the theory that allows one to relate $\kappa$-orbital integrals on a reductive algebraic group to stable orbital integrals on smaller endoscopic groups. It has been a key tool in establishing special cases of Langlands functoriality, which relates automorphic representations of such a group to its endoscopic friends. Endoscopy owes its existence to the following phenomenon: if $G$ is a connected reductive group over a field $F$ with algebraic closure $\bar{F}$, and we are given an $F$-representation $V$, then two elements $\gamma, \gamma^{\prime} \in V(F)$ may be $G(\bar{F})$ conjugate but not necessarily $G(F)$ conjugate. The $G(F)$ conjugacy classes in the $G(\bar{F})$-conjugacy class of $\gamma$ are parametrised by the pointed set $\mathcal{D}=\operatorname{ker}\left[H^{1}\left(F, I_{\gamma}\right) \rightarrow H^{1}(F, G)\right]$ where $I_{\gamma}$ is the centraliser of $\gamma$. In favourable circumstances such as the one in this paper, $\mathcal{D}$ is actually a finite abelian group, and in general one can replace it with an abelian group using the technology in [1]. If $V$ is the adjoint representation or a representation of a subgroup $H$ of $G$ on an $H$-invariant subspace of $V$ and $\gamma$ is semisimple with torus centraliser and regular with respect to this representation, then we say that $\gamma$ and $\gamma^{\prime}$ are stably conjugate whenever they are $G(\bar{F})$ conjugate. We assume that $\gamma$ is of this type for the rest of the paper since we will not actually need the more general definition of stable conjugacy.

If $F$ is now a complete nonarchimedean local field with ring of integers $\mathfrak{o}, G$ and $V$ are defined over $\mathfrak{o}$, and $\kappa: \mathcal{D} \rightarrow \mathbb{C}^{\times}$a character, we can form a $\kappa$-orbital integral, which is the sum of orbital integrals

$$
\mathcal{O}_{\gamma}^{\kappa}(\mathbf{1})=\sum_{\gamma^{\prime}} \kappa\left(\gamma^{\prime}\right) \int_{I_{\gamma^{\prime}}(F) \backslash G(F)} \mathbf{1}\left(\rho(g)^{-1} \gamma^{\prime}\right) \frac{\mathrm{d} g}{\mathrm{~d} t}
$$

(c) 2015 Polák; licensee Springer. This is an Open Access article distributed under the terms of the Creative Commons Attribution License (http://creativecommons.org/licenses/by/4.0), which permits unrestricted use, distribution, and reproduction in any medium, provided the original work is properly credited. 
where $\mathbf{1}$ is the characteristic function of $V(\mathfrak{o})$ and the Haar measures are chosen appropriately so that the integral points have unit volume. We have abused notation by writing $\kappa\left(\gamma^{\prime}\right)$ for $\kappa$ evaluated at the cohomology class corresponding to $\gamma^{\prime}$. In this special case of $\kappa=1$, this sum of integrals is called a stable orbital integral and is written $\mathcal{S O}_{\gamma}$. The fundamental lemma for Lie algebras, established in full generality by Ngô [5], states that there exists for each $\kappa$ a reductive algebraic group $H$ over $F$; and for each regular semisimple $\gamma \in \mathfrak{g}(F)$, there exists a regular semisimple $\gamma_{H} \in \mathfrak{h}(F)$ such that

$$
\mathcal{O}_{\gamma}^{\kappa}=\Delta\left(\gamma, \gamma_{H}\right) \mathcal{S O}_{\gamma_{H}}
$$

where the representation is the adjoint representation.

Here, $\Delta\left(\gamma, \gamma_{H}\right) \in \mathbb{C}$ is some factor that depends only on $\gamma$ and $\gamma_{H}$ and is in fact a power of $q$ up to a root of unity. This identity allows one to compare the stabilised trace formula on $G$ and on $H$, which has led to spectacular applications in establishing special cases of Langlands functoriality.

\section{In this paper}

We initiate the study of $\kappa$-orbital integrals for a pair $(G, \theta)$ where $G$ is a connected reductive algebraic group over a complete nonarchimedean local field and $\theta: G \rightarrow G$ is an involution, important for the theory of various relative trace formula. Now, instead of the adjoint representation, we use the adjoint action of the identity component of the $\theta$-fixed points, $\left(G^{\theta}\right)^{\circ}$ on $\mathfrak{g}_{1}=\{x \in \mathfrak{g}: \theta(x)=-x\}$. Hence, the group $G_{0}:=\left(G^{\theta}\right)^{\circ}$ is the focus of attention. In this setting, we have a $\kappa$-orbital integral

$$
\mathcal{O}_{\gamma}^{\kappa}(\mathbf{1})=\sum_{\gamma^{\prime}} \kappa\left(\gamma^{\prime}\right) \int_{I_{\gamma^{\prime}}(F) \backslash G_{0}(F)} \mathbf{1}\left(\operatorname{Ad}(g)^{-1} \gamma^{\prime}\right) \frac{\mathrm{d} g}{\mathrm{~d} t}
$$

We prove a fundamental lemma for $(\mathrm{U}(4), \theta)$ where $\theta$ is an involution such that $\mathrm{U}(4)^{\theta} \cong$ $\mathrm{U}(2) \times \mathrm{U}(2)$ and when $\gamma$ is of the form $\gamma=\operatorname{diag}(x, y,-y,-x)$ with $x \neq \pm y \in F^{\times}$. Motivated by the usual fundamental lemma for unitary groups, we define for a nontrivial $\kappa: \mathcal{D}\left(I_{\gamma}\right) \rightarrow \mathbb{C}^{\times}$the endoscopic symmetric space to be $\left(H, \theta_{H}\right)=\left(\mathrm{U}_{2}, \sigma\right) \times\left(\mathrm{U}_{2}, \sigma\right)$ where $\sigma: \mathrm{U}_{2} \rightarrow \mathrm{U}_{2}$ is such that $\mathrm{U}_{2}^{\sigma} \cong \mathrm{U}_{1} \times \mathrm{U}_{1}$. We then set $\gamma_{H}=\operatorname{diag}(x,-x) \times \operatorname{diag}(y,-y) \in \mathfrak{h}_{1}$. We assume $\gamma$ to be nearly singular, which by definition means that $v(x+y)>v(x-y)$. Under these assumptions, we prove the following fundamental lemma.

Theorem 1.2.1. The $\kappa$-orbital as defined in Equation 1 satisfies

$$
\mathcal{O}_{\gamma}^{\kappa}\left(\mathbf{1}_{\mathfrak{g}_{1}(\mathfrak{o})}\right)=\Delta\left(\gamma, \gamma_{H}\right) \mathcal{S} \mathcal{O}_{\gamma_{H}}\left(\mathbf{1}_{\mathfrak{h}_{1}(\mathfrak{o})}\right)
$$

where $\Delta\left(\gamma, \gamma_{H}\right) \in \mathbb{C}$ can be calculated explicitly and is a simple power of $q$ up to a root of unity.

Even though relative orbital integrals have been considered previously in the literature, this is the first known example of endoscopy in this setting and will be helpful in formulating more general relative endoscopic fundamental lemmas. Our primary motivation is the relative trace formula studied in [2], the original goal of those authors being to produce nontrivial cycles on unitary Shimura varieties. In that paper, the authors use the group analogue of the orbital integrals in this paper. Conjecturally, our Lie algebra version 
we study should say something about the group version of these orbital integrals, but as of yet a translation from one situation to the other is still an important open problem.

\section{Conventions and convenient facts}

Fields, groups and Haar measures

Let $F$ be a complete nonarchimedean local field of zero or odd positive characteristic with algebraic closure $\bar{F}$ and residue field $\mathbb{F}_{q}$. Let $E / F$ be an unramified quadratic extension of $F$. We denote the nontrivial action of the Galois group by $x \mapsto \bar{x}$. Since $E / F$ is fixed throughout, we simply use $N(x)=x \bar{x}$ to denote the norm of $x$. We fix once and for all a $\delta \in E$ such that $\bar{\delta}=-\delta$ and $v(\delta)=0$ so that $F \delta=\{x \in E: \bar{x}=-x\}$. Let $\mathfrak{o} \subset F$ be the ring of integers with maximal ideal $\mathfrak{m}$, and let $\mathfrak{o}_{E}$ be the ring of integers of $E$. We denote by $\pi$ a uniformizer of $F$, so that he resulting valuation $v: F^{\times} \rightarrow \mathbb{Z}$ is given by $v(\pi)=1$.

In our computations, we will consider various Haar measures on locally compact groups of the form $G(F)$ where $G$ is a linear algebraic group over $\mathfrak{o}$. These are always normalised so that $G(\mathfrak{o})$ has unit volume. We will need the following proposition which follows from $\mathfrak{o}_{E}$ being stable under $\operatorname{Gal}(E / F)$.

Proposition 2.1.1. If $a x+b \in \mathfrak{o}_{E}$ where $a, b \in F$ and $x \in F \delta$ then $a x \in \mathfrak{o}_{E}$ and $b \in \mathfrak{o}_{E}$.

\section{Symmetric space representations and unitary groups}

\section{Symmetric spaces}

In this section, we define the symmetric space representation and recall some facts we will need.

Definition 3.1.1. Let $\theta: G \rightarrow G$ be an involution of a reductive algebraic group over a field $F$. We abuse notation by writing $\theta$ also for the differential of $\theta$. We define the symmetric space representation to be the representation of $G_{0}=\left(G^{\theta}\right)^{\circ}$ on the-1-eigenspace $\mathfrak{g}_{1}=\{x \in \mathfrak{g}: \theta(x)=-x\}$.

The Lie subalgebra $\mathfrak{g}_{0}=\mathfrak{g}^{\theta}$ of fixed points also plays an important role. For $x \in \mathfrak{g}_{1}$, if $\operatorname{dim} \mathfrak{z}_{\mathfrak{g}_{0}}(x) \leq \operatorname{dim} \mathfrak{z}_{\mathfrak{g}_{0}}(y)$ for all $y \in \mathfrak{g}_{1}$, then we say that $x$ is regular. This is equivalent to the orbit $G_{0} \cdot x$ having minimal dimension. For this and further facts, the reader is referred to the paper [4].

\section{$\mathrm{U}_{2} \times \mathrm{U}_{2} \hookrightarrow \mathrm{U}_{4}$ and regular elements}

Recall that $F$ is a complete nonarchimedean local field of positive characteristic, and $E$ is an unramified quadratic extension (see 'Fields, groups and Haar measures'). Define the $n \times n$ matrix

$$
J_{n}=\left(\begin{array}{l}
1 \\
1
\end{array}\right) .
$$

The $n \times n$ unitary group functor is defined for all $F$-algebras $R$ by

$$
\mathrm{U}_{n}(R)=\left\{g \in \mathrm{GL}_{n}\left(R \otimes_{F} E\right): J_{n} \bar{g}^{-t} J_{n}=g\right\}
$$


and its Lie algebra is the functor given by

$$
\mathfrak{g}:=\mathfrak{u}_{n}(R)=\left\{x \in \mathfrak{g l}_{n}\left(R \otimes_{F} E\right):-J_{n} \bar{x}^{t} J_{n}=x\right\} .
$$

From now on, we consider the case $n=4$. We let $\theta: \operatorname{Res}_{E / F} \mathrm{GL}_{4} \rightarrow \operatorname{Res}_{E / F} \mathrm{GL}_{4}$ be conjugated by

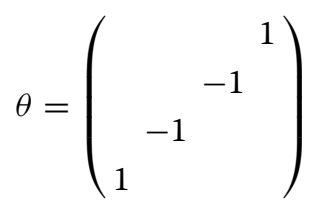

which by abuse of notation we also call $\theta$. Since $\theta J=J \theta$, the involution $\theta$ on $\operatorname{Res}_{E / F} \mathrm{GL}_{4}$ gives a well-defined involution on $\mathrm{U}_{4}$. For computational purposes, it is necessary to write down explicitly the $F$ points of the -1 -eigenspace $\mathfrak{g}_{1}$ in terms of matrices. This can be most easily done by observing that

$$
\left\{x \in \mathfrak{g l}_{4}(E): \theta(x)=-x\right\}=\left\{\left(\begin{array}{cccc}
x_{11} & x_{12} & x_{13} & x_{14} \\
x_{21} & x_{22} & x_{23} & x_{24} \\
x_{24} & -x_{23} & -x_{22} & x_{21} \\
-x_{14} & x_{13} & x_{12} & -x_{11}
\end{array}\right): x_{i j} \in E\right\}
$$

so that $\mathfrak{g}_{1}$ is then the fixed points of this under $x \mapsto-J_{4} \bar{x}^{t} J_{4}$ so that the $F$ points are

$$
\mathfrak{g}_{1}(F)=\left\{\left(\begin{array}{cccc}
x_{11} & x_{12} & x_{13} & x_{14} \\
-\bar{x}_{12} & x_{22} & x_{23} & -\bar{x}_{13} \\
-\bar{x}_{13} & -x_{23} & -x_{22} & -\bar{x}_{12} \\
-x_{14} & x_{13} & x_{12} & -x_{11}
\end{array}\right): \begin{array}{c}
x_{11}, x_{22} \in F \\
x_{14}, x_{23} \in F \delta
\end{array}\right\} .
$$

The fixed-point group $\mathrm{U}_{4}^{\theta} \cong \mathrm{U}_{2} \times \mathrm{U}_{2}=$ : $G_{0}$ acts on $\mathfrak{g}_{1}$. Inside $\mathfrak{g}_{1}(F)$ there is $\mathfrak{a}$, a maximal subspace of commuting semisimple elements such that

$$
\mathfrak{a}(F)=\left\{\left(\begin{array}{cccc}
x & 0 & 0 & 0 \\
0 & y & 0 & 0 \\
0 & 0 & -y & 0 \\
0 & 0 & 0 & -x
\end{array}\right): x, y \in F\right\} .
$$

We only consider regular semisimple elements in $\mathfrak{a}(F)$. If $\gamma=\operatorname{diag}(x, y,-y,-x) \in \mathfrak{a}(F)$, then $\gamma$ is regular if and only if $x, y \in F^{\times}$and $x \neq \pm y$. For any regular semisimple element $\gamma \in \mathfrak{g}_{1}(F)$ with centraliser $I_{\gamma}$ in $\mathrm{U}_{2} \times \mathrm{U}_{2}$ and for any compactly supported complex-valued smooth function $f$ on $\mathfrak{g}_{1}(F)$, we define the orbital integral

$$
\mathcal{O}_{\gamma}(f):=\int_{I_{\gamma}(F) \backslash \mathrm{U}_{2} \times \mathrm{U}_{2}(F)} f\left(\operatorname{Ad}\left(g^{-1}\right) \gamma\right) \frac{\mathrm{d} g}{\mathrm{~d} g_{\gamma}} .
$$

\section{Stable conjugacy}

Fix a regular $\gamma=\operatorname{diag}(x, y,-y,-x) \in \mathfrak{a}(F)$. The stable conjugacy class of $\gamma$ in $\mathfrak{g}_{1}(F)$ by definition is $G_{0}(\bar{F}) \gamma \cap \mathfrak{g}_{1}(F)$. When using cohomology, it is useful to express the stable conjugacy class of $\gamma$ as

$$
\left\{\operatorname{Ad}(g) \gamma: g \in G_{0}(\bar{F}) \text { and } g^{-1} \sigma(g) \in I_{\gamma}(\bar{F}) \text { for all } \sigma \in \operatorname{Gal}(\bar{F} / F)\right\} \text {. }
$$


In this section, we explicitly decompose the stable conjugacy class of $\gamma$ into $G_{0}(F)$ conjugacy classes. As before, we denote by $I_{\gamma}$ the stabiliser of $\gamma$ in $G_{0}$. The inclusion $I_{\gamma} \rightarrow G_{0}$ gives rise to a long exact sequence of pointed sets

$$
1 \rightarrow I_{\gamma}(F) \rightarrow G_{0}(F) \rightarrow\left(G_{0} / I_{\gamma}\right)(F) \rightarrow H^{1}\left(F, I_{\gamma}\right) \rightarrow H^{1}\left(F, G_{0}\right)
$$

in nonabelian cohomology. One easily checks that the map $\operatorname{Ad}(g) \gamma \mapsto\left(\sigma \mapsto g^{-1} \sigma(g)\right)$ is a well-defined bijection from the set of conjugacy classes of $\gamma$ in the stable conjugacy class to $\mathcal{D}:=\operatorname{ker}\left[H^{1}\left(F, I_{\gamma}\right) \rightarrow H^{1}\left(F, G_{0}\right)\right]$. Using this correspondence, we can compute explicit representatives for the conjugacy classes within the stable conjugacy class of $\gamma$.

We first compute

$$
I_{\gamma}=\left\{\left(\begin{array}{cccc}
a_{11} & 0 & 0 & 0 \\
0 & a_{22} & 0 & 0 \\
0 & 0 & a_{22} & 0 \\
0 & 0 & 0 & a_{11}
\end{array}\right): a_{i i} \bar{a}_{i i}=1\right\} \text {. }
$$

In other words, $I_{\gamma} \cong \mathrm{U}_{1} \times \mathrm{U}_{1}$ (which suggests our choice of an endoscopic symmetric space). We can compute the Galois cohomology over the finite extension $E / F$ which splits $I_{\gamma}$. Doing this, we find that

$$
H^{1}\left(F, I_{\gamma}\right) \cong \mathbb{Z} / 2 \times \mathbb{Z} / 2 .
$$

A quick application of Tate-Nakayama duality shows that the kernel $\mathcal{D}$ of $H^{1}\left(F, I_{\gamma}\right) \rightarrow$ $H^{1}\left(F, G_{0}\right)$ is $\mathbb{Z} / 2$. For the purposes of computations, let us be more explicit. Fix the isomorphism of algebras $E \otimes_{F} E \stackrel{\sim}{\rightarrow} E \oplus E$ given on pure tensors by $(a \otimes b) \mapsto(a b, \bar{a} b)$, where the multiplication on $E \oplus E$ is pointwise and the Galois action on the left factor of $E \otimes_{F} E$ translates to $\overline{(a, b)}=(b, a)$ in $E \oplus E$.

Let $\sigma \in \operatorname{Gal}(E / F)$ be the nontrivial element. The nontrivial element in $\mathcal{D}$ is then represented (for example) by the cocycle

$$
\sigma \mapsto\left(\pi I_{4}, \pi^{-1} I_{4}\right) \in I_{\gamma}(E)
$$

The element in $\left(I_{\gamma} \backslash G_{0}\right)(E)$ that maps to the corresponding cohomology class is represented by $(B, \pi B) \in G_{0}(E)$ where

$$
B=\frac{1}{2}\left(\begin{array}{cccc}
\pi^{-1}+1 & -\pi^{-1}+1 & 0 & 0 \\
-\pi^{-1}+1 & \pi^{-1}+1 & 0 & 0 \\
0 & 0 & \pi^{-1}+1 \pi^{-1}-1 \\
0 & 0 & \pi^{-1}-1 \pi^{-1}+1
\end{array}\right) .
$$

Indeed, it maps to $\sigma \mapsto g^{-1} \sigma(g)=\left(\pi I_{4}, \pi^{-1} I_{4}\right)$. We remark to the unwary reader that $\sigma$ here acts on the right of $E \otimes_{F} E$, which on $E \oplus E$ is the same $(a, b) \mapsto(\bar{b}, \bar{a})$. Now, $H^{1}\left(F, I_{\gamma}\right) \cong H^{1}\left(\mathbb{Z} / 2, I_{\gamma}(E)\right)$ and $I_{\gamma}(E) \cong\left\{\operatorname{diag}\left(\left(a, a^{-1}\right),\left(b, b^{-1}\right),\left(b, b^{-1}\right),\left(a, a^{-1}\right)\right)\right.$ : $\left.a, b \in E^{\times}\right\}$, so we are just computing group cohomology of a cyclic group. We get

$$
\begin{aligned}
H^{1}\left(\mathbb{Z} / 2, I_{\gamma}(E)\right) & \cong\left(E^{\times} /\left(E^{\times}\right)^{2}\right) \times\left(E^{\times} /\left(E^{\times}\right)^{2}\right) \\
& \cong \mathbb{Z} / 2 \times \mathbb{Z} / 2 .
\end{aligned}
$$


Hence, $\sigma \mapsto\left(\pi I_{4}, \pi^{-1} I_{4}\right)$ is a nontrivial cocycle because $\pi$ is not a square in $E$. We set $\gamma_{\text {stc }}=\operatorname{Ad}(B) \gamma$. Now let $\kappa: \mathcal{D} \rightarrow\{ \pm 1\}$ be a character. We have for any compactly supported smooth function $f$ on $\mathfrak{g}_{1}(F)$ the $\kappa$-orbital integral

$$
\mathcal{O}_{\gamma}^{\kappa}(f)=\int_{I_{\gamma}(F) \backslash G_{0}(F)} f\left(\operatorname{Ad}\left(g^{-1}\right) \gamma\right) \mathrm{d} g+\kappa(-1) \int_{I_{\gamma_{\mathrm{scc}}}(F) \backslash G_{0}(F)} f\left(\operatorname{Ad}\left(g^{-1}\right) \gamma_{\mathrm{stc}}\right) \mathrm{d} g .
$$

We note that we can omit the centralisers of $\gamma$ and of $\gamma_{\text {stc }}$ since their $F$ points are compact. We would like to compute this integral when $\kappa$ is the nontrivial character and when $f=\mathbf{1}$, the characteristic function of $\mathfrak{g}_{1}(\mathfrak{o})$ :

$$
\mathcal{O}_{\gamma}^{\kappa}(\mathbf{1})=\int_{G_{0}(F)} \mathbf{1}\left(\operatorname{Ad}\left(g^{-1}\right) \gamma\right) \mathrm{d} g-\int_{G_{0}(F)} \mathbf{1}\left(\operatorname{Ad}\left(g^{-1}\right) \gamma_{\text {stc }}\right) \mathrm{d} g .
$$

This is the goal of the remainder of the paper.

\section{Preliminaries on integration}

We choose a parabolic $P$ so that we get an Iwasawa decomposition $G_{0}(F)=P(F) G_{0}(\mathfrak{o})=$ $M(F) U(F) G_{0}(\mathfrak{o})$, where $M$ is a Levi subgroup of $P$ and $U$ is the unipotent radical of $P$; so we reduce the computation to one on $P(F)=M(F) U(F)$. Although this does simplify matters, since the centraliser of $\gamma$ is compact, we unfortunately cannot use the method of descent that would otherwise make the computation significantly easier. Now, to specify a parabolic of $G_{0}$ is the same thing as giving a cocharacter $\lambda: \mathbb{G}_{m} \rightarrow G_{0}$. We use the cocharacter

$$
\begin{array}{rl}
\mathbb{G}_{m} \longrightarrow G_{0} & r \longmapsto \frac{1}{2}\left(\begin{array}{cccc}
r+r^{-1} & 0 & -r+r^{-1} & 0 \\
0 & r+r^{-1} & 0 & r-r^{-1} \\
-r+r^{-1} & 0 & r+r^{-1} & 0 \\
0 & r-r^{-1} & 0 & r+r^{-1}
\end{array}\right) .
\end{array}
$$

One verifies easily that $\lambda$ is both $\theta$ fixed and actually does land in $\mathrm{U}_{4}$. This cocharacter uniquely specifies the parabolic $P=\left\{g \in G_{0}: \lim _{r \rightarrow 0} \lambda(r) g \lambda\left(r^{-1}\right)\right.$ exists $\}$. The unipotent radical of this parabolic is given by $U=\left\{g \in G_{0}: \lim _{r \rightarrow 0} \lambda(r) g \lambda\left(r^{-1}\right)=1\right\}$, and we calculate the $F$ points of the unipotent radical to be isomorphic to $F \delta \times F \delta$ via

$$
\begin{aligned}
F \delta \times F \delta & \longrightarrow U(F) \\
\quad(c, d) \longmapsto & \left(\begin{array}{cccc}
c+1 & d & c & -d \\
-d & -c+1 & -d & c \\
-c & -d & -c+1 & d \\
-d & -c & -d & c+1
\end{array}\right) .
\end{aligned}
$$

For integrating, we use the product Haar measure on $F \delta \times F \delta$ where on each factor $F \delta$ we choose a Haar measure so that $o \delta$ has unit volume. A Levi subgroup of $P$ is the subgroup that centralises the cocharacter $\lambda$. We calculate its $F$ points to be isomorphic to $E^{\times} \times E^{\times}$through the isomorphism

$$
\begin{aligned}
E^{\times} \times E^{\times} \longrightarrow M(F) \\
\left(r_{1}, r_{2}\right) \longmapsto \frac{1}{4}\left(\begin{array}{cccc}
r_{1}+r_{2}+\bar{r}_{1}^{-1}+\bar{r}_{2}^{-1} & r_{1}+r_{2}-\bar{r}_{1}^{-1}-\bar{r}_{2}^{-1} & r_{1}-r_{2}-\bar{r}_{1}^{-1}+\bar{r}_{2}^{-1} & -r_{1}+r_{2}-\bar{r}_{1}^{-1}+\bar{r}_{2}^{-1} \\
r_{1}+r_{2}-\bar{r}_{1}^{-1}-\bar{r}_{2}^{-1} & r_{1}+r_{2}+\bar{r}_{1}^{-1}+\bar{r}_{2}^{-1} & r_{1}-r_{2}+\bar{r}_{1}^{-1}-\bar{r}_{2}^{-1} & -r_{1}+r_{2}+\bar{r}_{1}^{-1}-\bar{r}_{2}^{-1} \\
r_{1}-r_{2}-\bar{r}_{1}^{-1}+\bar{r}_{2}^{-1} & r_{1}-r_{2}+\bar{r}_{1}^{-1}-\bar{r}_{2}^{-1} & r_{1}+r_{2}+\bar{r}_{1}^{-1}+\bar{r}_{2}^{-1} & -r_{1}-r_{2}+\bar{r}_{1}^{-1}+\bar{r}_{2}^{-1} \\
-r_{1}+r_{2}-\bar{r}_{1}^{-1}+\bar{r}_{2}^{-1} & -r_{1}+r_{2}+\bar{r}_{1}^{-1}-\bar{r}_{2}^{-1} & -r_{1}-r_{2}+\bar{r}_{1}^{-1}+\bar{r}_{2}^{-1} & r_{1}+r_{2}+\bar{r}_{1}^{-1}+\bar{r}_{2}^{-1}
\end{array}\right)
\end{aligned}
$$


Again, for integration, we use the product Haar measure on $E^{\times} \times E^{\times}$so that $\mathfrak{o}_{E}^{\times}$in $E^{\times}$ has unit volume.

We note that multiplying the matrix in Equation 4 either on the left or the right by the matrix that represents the cocycle is the same matrix but with $\bar{r}_{i}$ replaced with $\pi \bar{r}_{i}$ for $i=1,2$. Thus, in any expressions that are a function of $m \gamma m^{-1}$ for $m \in M$, making this replacement gives us the equations for $m \gamma_{\mathrm{stc}} m^{-1}$.

In making these reductions, we are left to evaluate the integral

$$
\begin{aligned}
\mathcal{O}_{\gamma}^{\kappa}(\mathbf{1})= & \int_{M(F)} \int_{U(F)} \mathbf{1}\left(\operatorname{Ad}\left(u^{-1}\right) \operatorname{Ad}\left(m^{-1}\right) \gamma\right) \mathrm{d} u \mathrm{~d} m \\
& -\int_{M(F)} \int_{U(F)} \mathbf{1}\left(\operatorname{Ad}\left(u^{-1}\right) \operatorname{Ad}\left(m^{-1}\right) \gamma_{\mathrm{stc}}\right) \mathrm{d} u \mathrm{~d} m
\end{aligned}
$$

where the Haar measures are chosen so that $U(\mathfrak{o})$ and $M(\mathfrak{o})$ each have unit volume. We now examine an element of the form $Y=\operatorname{Ad}\left(u^{-1}\right) \operatorname{Ad}\left(m^{-1}\right) \gamma$, where $u$ is a matrix as in Equation 3 and $m$ is a matrix as in Equation 4. Since $Y \in \mathfrak{g}_{1}$, by the explicit form of $\mathfrak{g}_{1}$ in Equation 2, we see that $Y \in \mathfrak{g}_{1}(\mathfrak{o})$ exactly when $v=\left[Y_{11}, Y_{12}, Y_{13}, Y_{14}, Y_{22}, Y_{23}\right]^{t} \in \mathfrak{o}^{6}$. And, $v \in \mathfrak{o}^{6}$ exactly when $A v \in \mathfrak{o}^{6}$ for any $A \in \mathrm{GL}_{6}(\mathfrak{o})$. In particular, we take

$$
A=\left(\begin{array}{cccccc}
0 & -1 & 1 & 0 & -1 & 1 \\
1 & -1 & -1 & -1 & 0 & 0 \\
0 & 1 & 1 & 0 & -1 & -1 \\
1 & 1 & -1 & 1 & 0 & 0 \\
1 & 1 & 1 & -1 & 0 & 0 \\
1 & -1 & 1 & 1 & 0 & 0
\end{array}\right), \quad A v=\left(\begin{array}{c}
-Y_{12}+Y_{13}-Y_{22}+Y_{23} \\
Y_{11}-Y_{12}-Y_{13}-Y_{14} \\
Y_{12}+Y_{13}-Y_{22}-Y_{23} \\
Y_{11}+Y_{12}-Y_{13}+Y_{14} \\
Y_{11}+Y_{12}+Y_{13}-Y_{14} \\
Y_{11}+Y_{13}-Y_{12}+Y_{14}
\end{array}\right) .
$$

We calculate that $\operatorname{det}(A)=-32$, so that indeed $A \in \mathrm{GL}_{6}(\mathfrak{o})$. Now, it is simply a matter of calculating $Y=\operatorname{Ad}\left(u^{-1}\right) \operatorname{Ad}\left(m^{-1}\right) \gamma$ and each of the quantities in $A v$. Straightforward but tedious computations and making the harmless change of variables $(d-c \rightsquigarrow c, d+c \rightsquigarrow d)$ show that

$$
\begin{aligned}
-Y_{12}+Y_{13}-Y_{22}+Y_{23} & =\frac{r_{2}}{r_{1}}(x+y)\left(-c-\frac{1}{2}\right)+\frac{1}{2}(x-y) \frac{1}{r_{1} \bar{r}_{2}} \\
\bar{Y}_{11}-\bar{Y}_{12}-\bar{Y}_{13}-\bar{Y}_{14}= & \frac{r_{2}}{r_{1}}(x+y)\left(-c+\frac{1}{2}\right)+\frac{1}{2}(x-y) \frac{1}{r_{1} \bar{r}_{2}} \\
\bar{Y}_{12}+\bar{Y}_{13}-\bar{Y}_{22}-\bar{Y}_{23}= & \frac{r_{2}}{r_{1}}(x+y)\left(-d-\frac{1}{2}\right)+\frac{1}{2}(x-y) \bar{r}_{1} r_{2} \\
Y_{11}+Y_{12}-Y_{13}+Y_{14}= & \frac{r_{2}}{r_{1}}(x+y)\left(-d+\frac{1}{2}\right)+\frac{1}{2}(x-y) \bar{r}_{1} r_{2} \\
\bar{Y}_{11}+\bar{Y}_{12}+\bar{Y}_{13}-\bar{Y}_{14} & =2 \frac{r_{2}}{r_{1}} c d(x+y)-d\left[\frac{r_{2}}{r_{1}}(x+y)+\frac{1}{r_{1} \bar{r}_{2}}(x-y)\right] \\
& -\bar{r}_{1} r_{2} c(x-y)+\frac{1}{2}\left[\bar{r}_{1} r_{2}(x-y)+\frac{\bar{r}_{1}}{\bar{r}_{2}}(x+y)\right] \\
Y_{11}+Y_{13}-Y_{12}+Y_{14}= & 2 \frac{r_{2}}{r_{1}} c d(x+y)-c\left[\frac{r_{2}}{r_{1}}(x+y)+\bar{r}_{1} r_{2}(x-y)\right] \\
- & \frac{1}{r_{1} \bar{r}_{2}} d(x-y)+\frac{1}{2}\left[\frac{\bar{r}_{1}}{\bar{r}_{2}}(x+y)+\frac{1}{r_{1} \bar{r}_{2}}(x-y)\right]
\end{aligned}
$$

We note that we have also harmlessly replaced some terms by their conjugates. We now simplify these terms even further, preserving their status of integrality. Subtracting 
Equation 5 from Equation 6 shows that the integrality of these implies that $\frac{r_{2}}{r_{1}}(x+y)$ is integral. In particular, it thus follows that if Equation 5 is integral then so is

$$
\frac{r_{2}}{r_{1}}(x+y)(-c)+\frac{1}{2}(x-y) \frac{1}{r_{1} \bar{r}_{2}} \text {. }
$$

Multiplying this expression by the valuation-zero term $\pi^{-\left(v\left(r_{1}\right)+v\left(r_{2}\right)\right)} r_{1} \bar{r}_{2}$ and applying Proposition 2.1.1 shows that each term of this expression is in fact integral exactly when the entire expression is integral. We can of course apply the same reasoning to Equations 7 and 8, which shows that Equation 5 to Equation 8 being integral is equivalent to the following expressions being integral:

$$
\begin{array}{ll}
E_{1}=\frac{r_{2}}{r_{1}}(x+y) & E_{4}=(x-y) \frac{1}{r_{1} \bar{r}_{2}} \\
E_{2}=\frac{r_{2}}{r_{1}}(x+y) c & E_{5}=(x-y) \bar{r}_{1} r_{2} \\
E_{3}=\frac{r_{2}}{r_{1}}(x+y) d &
\end{array}
$$

We observe also that if we subtract Equation 8 from Equation 9, we get the same thing as subtracting Equation 6 from Equation 10, both differences being equal to

$$
2 \frac{r_{2}}{r_{1}} c d(x+y)-(x-y)\left[c \bar{r}_{1} r_{2}+\frac{d}{r_{1} \bar{r}_{2}}\right]+\frac{1}{2} \frac{\bar{r}_{1}}{\bar{r}_{2}}(x+y)
$$

so we might as well replace Equations 9 and 10 by Equation 12 . We can again apply Proposition 2.1.1 to Equation 12 multiplied by $\pi^{-\left(v\left(r_{1}\right)+v\left(r_{2}\right)\right)} r_{1} \bar{r}_{2}$, which tells us that the integrality of Equation 12 is actually equivalent to the integrality of these two:

$$
\begin{aligned}
& E_{6}=4 \frac{r_{2}}{r_{1}} c d(x+y)+\frac{\bar{r}_{1}}{\bar{r}_{2}}(x+y) \\
& E_{7}=(x-y)\left[c \bar{r}_{1} r_{2}+\frac{d}{r_{1} \bar{r}_{2}}\right]
\end{aligned}
$$

We have come to the end of our simplifications on the conditions that determine whether $Y=\operatorname{Ad}\left(u^{-1}\right) \operatorname{Ad}\left(m^{-1}\right) \gamma$ is integral.

\section{Elimination of fiendish cases}

We have determined in 'Preliminaries on integration' the expressions $E_{1}, E_{2}, \ldots, E_{7}$ that are integral exactly when $\operatorname{Ad}\left(u^{-1}\right) \operatorname{Ad}\left(m^{-1}\right) \gamma$ is integral. We denote by $E_{1}^{\text {stc }}, E_{2}^{\text {stc }}, \ldots, E_{7}^{\text {stc }}$ the corresponding equations for $\gamma_{\text {stc }}$. Recall that to get the conditions for $\gamma_{\text {stc }}$, we just replace $\bar{r}_{i}$ by $\pi \bar{r}_{i}$ for $i=1,2$ in $E_{1}, \ldots, E_{7}$. For the remainder of the paper, we set

$$
\begin{aligned}
h & =v\left(r_{1}\right)+v\left(r_{2}\right), \\
V_{m} & =v(x-y) \\
V_{p} & =v(x+y) .
\end{aligned}
$$


To aid the reader, we list $E_{1}, \ldots, E_{7}$ and their stably conjugate versions, along with their valuations:

$$
\begin{aligned}
& E_{1}=\frac{r_{2}}{r_{1}}(x+y) \\
& E_{1}^{\mathrm{stc}}=\frac{r_{2}}{r_{1}}(x+y) \\
& v\left(E_{1}\right)=h+V_{p}-2 v\left(r_{1}\right) \\
& E_{2}=\frac{r_{2}}{r_{1}}(x+y) c \\
& v\left(E_{2}\right)=h+V_{p}+v(c)-2 v\left(r_{1}\right) \\
& E_{3}=\frac{r_{2}}{r_{1}}(x+y) d \\
& v\left(E_{3}\right)=h+V_{p}+v(d)-2 v\left(r_{1}\right) \\
& E_{4}=(x-y) \frac{1}{r_{1} \bar{r}_{2}} \\
& v\left(E_{4}\right)=V_{m}-h \\
& E_{5}=(x-y) \bar{r}_{1} r_{2} \\
& v\left(E_{5}\right)=V_{m}+h \\
& E_{6}=4 \frac{r_{2}}{r_{1}} c d(x+y)+\frac{\bar{r}_{1}}{\overline{r_{2}}}(x+y) \\
& v\left(E_{6}\right) \geq \min \left\{\begin{array}{c}
h+V_{p}+v(c)+v(d)-2 v\left(r_{1}\right), \\
V_{p}-h+2 v\left(r_{1}\right)
\end{array}\right\} \quad v\left(E_{6}^{\mathrm{stc}}\right) \geq \min \left\{\begin{array}{c}
h+V_{p}+v(c)+v(d)-2 v\left(r_{1}\right), \\
V_{p}-h+2 v\left(r_{1}\right)
\end{array}\right\} \\
& E_{7}=(x-y)\left[c \bar{r}_{1} r_{2}+\frac{d}{r_{1} \bar{r}_{2}}\right] \\
& E_{7}^{\mathrm{stc}}=(x-y)\left[c \pi \bar{r}_{1} r_{2}+\frac{d}{\pi r_{1} \bar{r}_{2}}\right] \\
& v\left(E_{7}\right) \geq \min \left\{\begin{array}{l}
V_{m}+v(c)+h, \\
V_{m}+v(d)-h
\end{array}\right\} \\
& v\left(E_{1}^{\mathrm{stc}}\right)=h+V_{p}-2 v\left(r_{1}\right) \\
& E_{2}^{\mathrm{stc}}=\frac{r_{2}}{r_{1}}(x+y) c \\
& v\left(E_{2}^{\mathrm{stc}}\right)=h+V_{p}+v(c)-2 v\left(r_{1}\right) \\
& E_{3}^{\text {stc }}=\frac{r_{2}}{r_{1}}(x+y) d \\
& v\left(E_{3}^{\mathrm{stc}}\right)=h+V_{p}+v(d)-2 v\left(r_{1}\right) \\
& E_{4}^{\mathrm{stc}}=(x-y) \frac{1}{\pi r_{1} \bar{r}_{2}} \\
& v\left(E_{4}^{\mathrm{stc}}\right)=V_{m}-h-1 \\
& E_{5}^{\mathrm{stc}}=(x-y) \pi \bar{r}_{1} r_{2} \\
& v\left(E_{5}\right)=V_{m}+h+1 \\
& E_{6}^{\mathrm{stc}}=4 \frac{r_{2}}{r_{1}} c d(x+y)+\frac{\bar{r}_{1}}{\overline{r_{2}}}(x+y) \\
& v\left(E_{7}^{\mathrm{stc}}\right) \geq \min \left\{\begin{array}{l}
V_{m}+v(c)+h+1 \\
V_{m}+v(d)-h-1
\end{array}\right\}
\end{aligned}
$$

We see that only the fourth, fifth and seventh expressions differ between $\gamma$ and $\gamma_{\text {stc. }}$. We observe that the difficulties will occur mainly with the sixth and seventh, since they are sums. In this section, we eliminate some of the more fiendish difficulties to prepare the way for the main calculation in 'Brute force calculations' section. In order to lessen the wordiness and symbolism in the sequel, the summands of $E_{6}$ will refer to the two terms $4 \frac{r_{2}}{r_{1}} c d(x+y)$ and $\frac{\bar{r}_{1}}{\bar{r}_{2}}(x+y)$. Similarly, the summands of $E_{7}$ will refer to the two terms: $(x-y) c \bar{r}_{1} r_{2}$ and $(x-y) \frac{d}{r_{1} \bar{r}_{2}}$. We also use this terminology, suitably modified, for the stably conjugate versions. For example, if $E_{6}$ is integral, then we know that either both summands are integral or neither are. These possibilities for $E_{6}$ and $E_{7}$ break down the computation into various cases, and the next proposition shows that the worst of these actually cannot occur.

Lemma 3.5.1. If none of the summands in $E_{6}$ and $E_{7}$ are integral, then $E_{6}$ and $E_{7}$ cannot be simultaneously integral.

Proof. We proceed by contradiction, assuming that none of the summands in $E_{6}$ and $E_{7}$ are integral, but that both $E_{6}$ and $E_{7}$ are integral. In this case, the valuation of the first summand must be equal to the valuation of the second in $E_{6}$, and the same is true of $E_{7}$. Thus, we get a pair of equations:

$$
\begin{aligned}
& 2\left[v\left(r_{2}\right)-v\left(r_{1}\right)\right]+v(c)+v(d)=0, \\
& 2\left[v\left(r_{2}\right)+v\left(r_{1}\right)\right]+v(c)-v(d)=0 .
\end{aligned}
$$


In particular, $v(c)=-2 v\left(r_{2}\right)$ and $v(d)=2 v\left(r_{1}\right)$. Hence,

$$
v\left(\frac{r_{2}}{r_{1}} d(x+y) \pi^{-\left(h+V_{p}+V_{m}\right)}\right)=-V_{m} .
$$

Multiply $E_{6}$ by the inverse of the expression in $v(-)$ to get

$$
4 c \pi^{h+V_{p}+V_{m}}+\frac{N\left(r_{1}\right) \pi^{h+V_{p}+V_{m}}}{d N\left(r_{2}\right)} \in \pi^{V_{m}} \mathfrak{o} .
$$

Using the same procedure on $E_{7}$ gives

$$
4 c \pi^{h+V_{m}+V_{p}}+\frac{4 d \pi^{h+V_{m}+V_{p}}}{N\left(r_{1}\right) N\left(r_{2}\right)} \in \pi^{V_{p}} \mathfrak{o} .
$$

We take the difference between Equation 13 and Equation 14, obtaining

$$
\frac{\pi^{h+V_{m}+V_{p}}}{d N\left(r_{1}\right) N\left(r_{2}\right)}\left(N\left(r_{1}\right)-2 d\right)\left(N\left(r_{1}\right)+2 d\right) .
$$

We note that $N\left(r_{1}\right) \in F$ whereas $2 d \in F \delta$. Hence, the valuation of $N\left(r_{1}\right) \pm 2 d$ is precisely $v(d)$. Thus, the valuation of Equation 15 is $V_{m}+V_{p}+v(d)-h$. There are two cases to consider: either $V_{p} \geq V_{m}$ or $V_{p}<V_{m}$.

Case 1. $V_{p} \geq V_{m}$. Then, Equation 15 lies in $\pi^{V_{m}} \mathfrak{o}$, or in other words, $v(d)+V_{p}+V_{m}-$ $h \geq V_{m}$. Simplifying, we get $v(d)+V_{p}-h \geq 0$. On the other hand, the first summand of $E_{6}$ also has valuation $v(d)+V_{p}-h$, showing that this summand is integral, which is a contradiction.

Case 2. $V_{p}<V_{m}$. Then Equation 15 lies in $\pi^{V_{p}} \mathfrak{o}$, or in other words, $v(d)+V_{p}+V_{m}-h \geq$ $V_{p}$. Hence, $v(d)+V_{m}-h \geq 0$, but this is the valuation of the second summand of $E_{7}$, which is again a contradiction.

Remark 3.5.2. In this paper, case 2 in the above proof does not actually occur since we will assume for the actual computation that $V_{p}>V_{m}$, but we have included the more general statement for completeness.

The reader will have no trouble applying the same argument to prove the stably conjugate version.

Lemma 3.5.3. If none of the summands in $E_{6}^{\mathrm{stc}}$ and $E_{7}^{\mathrm{stc}}$ are integral, then $E_{6}^{\mathrm{stc}}$ and $E_{7}^{\mathrm{stc}}$ cannot be simultaneously integral.

The next lemma allows us a significant simplification if we stick with a 'limiting case' for $\gamma$.

Lemma 3.5.4. Suppose that $V_{p}>V_{m}$, that $E_{1}, E_{2}, \ldots, E_{7}$ are integral, and that not all the $E_{i}^{\text {stc }}$ are integral (the last condition being equivalent to: $E_{7}^{\text {stc }}$ is not integral). Under these conditions, if the summands of $E_{7}$ are integral, then the summands of $E_{6}$ are integral as well. 
Proof. We suppose by contradiction that we have a solution that makes $E_{1}$ to $E_{7}$ integral, that the summands of $E_{7}$ are integral, but that the summands of $E_{6}$ are not integral. Before we list the inequalities in this case, let us make three observations.

1. From $E_{2}$, we get $h+V_{p}+v(c)-2 v\left(r_{1}\right) \geq 0$. However, the first summand of $E_{6}$ not being integral is equivalent to $h+V_{p}+v(c)+v(d)-2 v\left(r_{1}\right)<0$. Hence, $v(d)<0$. Repeating the argument with $E_{3}$ in place of $E_{2}$ shows $v(c)<0$.

2. The valuation of the first summand of $E_{6}$ is equal to the valuation of the second. This implies that $v(c)+v(d)=4 v\left(r_{1}\right)-2 h$.

3. Since $E_{4}=E_{4}^{\text {stc }}$, any solution of $E_{1}, \ldots, E_{7}$ will also be a solution of the stably conjugate versions unless $v(d)=h-V_{m}$; so we evaluate only under this additional condition, and this implies based on our second observation that $v(c)=4 v\left(r_{1}\right)-3 h+V_{m}$.

Hence, we have the following inequalities, by using the substitutions in Equation 1 to Equation 3 in $E_{2}, E_{3}$, either term of $E_{6}$, and the first term of $E_{7}$ being integral:

$$
\begin{aligned}
2 v\left(r_{1}\right) & \geq 2 h-V_{p}-V_{m} \\
2 h+V_{p}-V_{m} & \geq 2 v\left(r_{1}\right) \\
h-V_{p} & >2 v\left(r_{1}\right) \\
2 v\left(r_{1}\right) & \geq h-V_{m}
\end{aligned}
$$

Or, more succinctly,

$$
\min \left\{2 h+V_{p}-V_{m}, h-V_{p}-1\right\} \geq 2 v\left(r_{1}\right) \geq \max \left\{2 h-V_{p}-V_{m}, h-V_{m}\right\} .
$$

We see that $2 h+V_{p}-V_{m} \geq h-V_{p}-1$ is equivalent to $h+2 V_{p}-V_{m}+1 \geq 0$. Since $V_{p}>V_{m}$, so that $h+2 V_{p}-V_{m}+1 \geq h+V_{m}+1 \geq 1$. Similarly, $h-V_{m} \geq 2 h-V_{m}-V_{m}$ is equivalent to $V_{p} \geq h$, which is true again since $V_{p}>V_{m}$. Hence, we have that

$$
h-V_{p}-1 \geq 2 v\left(r_{1}\right) \geq h-V_{m} .
$$

So $h-V_{p}-1 \geq h-V_{m}$ or, equivalently, $V_{m} \geq V_{p}+1$, which is absurd.

Again, the same argument will apply to the stably conjugate version.

Lemma 3.5.5. Suppose that $V_{p}>V_{m}$, and that $E_{1}^{\mathrm{stc}}, E_{2}^{\mathrm{stc}}, \ldots, E_{7}^{\mathrm{stc}}$ are integral, but that at least one of $E_{1}, \ldots, E_{7}$ is not integral. If the summands of $E_{7}^{\mathrm{stc}}$ are integral, then the summands of $E_{6}^{\mathrm{stc}}$ are integral as well.

\section{Brute force calculations}

\section{Preliminaries}

We introduced in the 'Symmetric space representations and unitary groups' section the orbital integral

$$
\mathcal{O}_{\gamma}^{\kappa}=\int_{G_{0}(F)} \mathbf{1}\left(\operatorname{Ad}(g)^{-1} \gamma\right) \mathrm{d} g-\int_{G_{0}(F)} \mathbf{1}\left(\operatorname{Ad}(g)^{-1} \gamma_{\text {stc }}\right) \mathrm{d} g .
$$

The integral $\int_{G_{0}(F)} \mathbf{1}\left(\operatorname{Ad}(g)^{-1} \gamma\right) \mathrm{d} g$ is the same as the measure of the set

$$
\left\{\left(c, d, r_{1}, r_{2}\right) \in F \delta \times F \delta \times E^{\times} \times E^{\times}: E_{1}, \ldots, E_{7} \text { are integral }\right\},
$$


and the analogous statement holds for the stably conjugate version. To evaluate the orbital integral, we do as follows: first, we find the measure of the subset of $F \delta \times F \delta \times E^{\times} \times E^{\times}$ such that all the $E_{*}$ are integral but at least one of $E_{*}^{\text {stc }}$ is not integral. We refer to this as the $(1,0)$ case. Similarly, the $(0,1)$ case is when all of $E_{*}^{\text {stc }}$ are integral but at least one of $E_{*}$ is not. We then take the measure of the $(1,0)$ case and subtract the measure of the $(0,1)$ case.

In this section, we carry out the calculation, evaluating the integral. Our strategy is to fix $h=v\left(r_{1}\right)+v\left(r_{2}\right)$, write down an expression for the measure of the solution set and then sum over all the possibilities for $h$ :

Lemma 4.1.1. The integrality of $E_{4}$ and $E_{5}$ is equivalent to the inequality $V_{m} \geq h \geq$ $-V_{m}$. Similarly, the integrality of $E_{4}^{\text {stc }}$ and $E_{5}^{\text {stc }}$ is equivalent to $V_{m}-1 \geq h \geq-V_{m}-1$. In either case, if $V_{m}<0$, then neither of this equalities can be satisfied and hence the $\kappa$-orbital integral vanishes.

Definition 4.1.2. We say that $\gamma$ is nearly singular if $V_{p}>V_{m}$.

For the rest of this paper, we assume that $\gamma$ is nearly singular, which is relatively harmless since our calculation under this assumption still should give us the correct transfer factor, assuming that there is a sane version of endoscopy operating in the midst. At any rate, in view of Lemmas 3.5.1, 3.5.3, 3.5.4 and 3.5.5, we then have to consider two possibilities: all summands in $E_{6}$ and in $E_{7}$ are integral, and the summands of $E_{6}$ are integral but the summands of $E_{7}$ are not.

\section{Integer arithmetic}

Here, we state the properties of floor and ceiling functions we use. For any $r \in \mathbb{R}$, we write $\lfloor r\rfloor$ and $\lceil r\rceil$ for the floor of $r$ and the ceiling of $r$, respectively. If $a, b \in \mathbb{Z}$, then we will frequently need the following facts that are easy to verify but included for convenience in following lengthy computations:

$$
\begin{aligned}
& |\{x \in \mathbb{Z}: a \geq 2 x \geq b\}|=\left\lfloor\frac{a}{2}\right\rfloor-\left\lceil\frac{b}{2}\right\rceil+1= \begin{cases}\frac{a-b}{2}+1 & \text { if } a, b \text { are even } \\
\frac{a-b}{2} & \text { if } a, b \text { are odd } \\
\frac{a-b+1}{2} & \text { if } a, b \text { have opposite parity }\end{cases} \\
& \left\lfloor\frac{a}{2}\right\rfloor=\left\lceil\frac{a-1}{2}\right\rceil \\
& \left\lfloor\frac{a}{2}+1\right\rfloor=\left\lfloor\frac{a+2}{2}\right\rfloor \\
& \left\lceil\frac{a}{2}\right\rceil+1=\left\lceil\frac{a+2}{2}\right\rceil
\end{aligned}
$$

\section{Measures}

In all sections, $(c, d) \in F \delta \times F \delta$, and $F \delta$ has the Haar measure so that $\mathfrak{o} \delta$ has unit volume. Then, $F \delta \times F \delta$ has the product measure. Moreover, $r_{1} \in E^{\times}$, and $E^{\times}$has the Haar measure so that $\mathfrak{o}_{E}^{\times}$has unit volume.

\section{All summands integral}

In this section, we evaluate case $(1,0)$ (resp. $(0,1))$ when all summands of $E_{6}$ and $E_{7}$ (resp. $E_{6}^{\text {stc }}$ and $\left.E_{7}^{\text {stc }}\right)$ are integral. We consider two cases: one where $h=V_{m}-1, \ldots,-V_{m}$ for 
both integrals, and the other where $h=V_{m}$ for $\mathcal{O}_{\gamma}$ and $h=-V_{m}-1$ for $\mathcal{O}_{\gamma_{\text {stc }}}$. In order to make reading this section easier, here are the inequalities that must be satisfied in this case:

Lemma 4.4.1. Suppose all summands are integral. Then, the inequalities defining the set that we must determine the measure of are:

$$
\begin{aligned}
& \text { For } \mathcal{O}_{\gamma} \quad \text { For } \mathcal{O}_{\gamma_{\text {stc }}} \\
& h+V_{p}-2 v\left(r_{1}\right) \geq 0 \\
& h+V_{p}+v(c)-2 v\left(r_{1}\right) \geq 0 \\
& h+V_{p}+v(d)-2 v\left(r_{1}\right) \geq 0 \quad \text { Same as for } \mathcal{O}_{\gamma} \\
& h+V_{p}+v(c)+v(d)-2 v\left(r_{1}\right) \geq 0 \\
& V_{p}-h+2 v\left(r_{1}\right) \geq 0 \\
& V_{m}+v(c)+h \geq 0 \\
& V_{m}+v(c)+h+1 \geq 0 \\
& V_{m}+v(d)-h \geq 0 \\
& V_{m}+v(d)-h-1 \geq 0
\end{aligned}
$$

Proof. We take the terms listed at the beginning of 'Elimination of fiendish cases', set the valuation of each of them to be greater than or equal to zero for $E_{1}$ to $E_{5}$ and $E_{1}^{\text {stc }}$ to $E_{5}^{\text {stc }}$ and set the valuation of each summand to be greater than or equal to zero for $E_{6}, E_{7}, E_{6}^{\text {stc }}$ and $E_{7}^{\text {stc }}$. We note that we have not written down the inequalities for $E_{4}, E_{4}^{\text {stc }}, E_{5}$ or $E_{5}^{\text {stc }}$ because these will automatically be integral given our assumptions on $h$.

Case $(1,0)$ : the integral $\mathcal{O}_{\gamma}$ for $h=V_{m}$

Our starting inequalities at the start of 'All summands integral' reduce to the following:

$$
\begin{aligned}
V_{m}+V_{p}-2 v\left(r_{1}\right) & \geq 0 \\
V_{m}+V_{p}+v(c)-2 v\left(r_{1}\right) & \geq 0 \\
V_{m}+V_{p}+v(d)-2 v\left(r_{1}\right) & \geq 0 \\
V_{m}+V_{p}+v(c)+v(d)-2 v\left(r_{1}\right) & \geq 0 \\
V_{p}-V_{m}+2 v\left(r_{1}\right) & \geq 0 \\
2 V_{m}+v(c) & \geq 0 \\
v(d) & \geq 0
\end{aligned}
$$

We note that since $h=V_{m}$, the expressions $E_{*}^{\text {stc }}$ cannot all be integral since in that case we must have $V_{m}-1 \geq h$. Since $v(d) \geq 0$, we see that Equations 19 and 20 are redundant, so we can eliminate them. In subsequent calculations, we shall frequently eliminate the obvious redundant inequalities without note. There are two cases to consider: $v(c) \geq 0$ and $v(c)<0$.

Case 1. $v(c) \geq 0$. The remaining inequalities are

$$
\begin{aligned}
V_{m}+V_{p} \geq 2 v\left(r_{1}\right) & \geq V_{m}-V_{p} \\
v(c) & \geq 0 \\
v(d) & \geq 0 .
\end{aligned}
$$


At this point, the reader may wish to review 'Integer arithmetic' containing various identities with floor and ceiling functions. Using these, we see that the measure of the corresponding set of solutions is

$$
\begin{cases}V_{p}+1 & \text { if } V_{m}+V_{p} \text { is even } \\ V_{p} & \text { if } V_{m}+V_{p} \text { is odd }\end{cases}
$$

Case 2. $v(c)<0$. Now the relevant inequalities are

$$
\begin{aligned}
V_{m}+V_{p}+v(c) \geq 2 v\left(r_{1}\right) & \geq V_{m}-V_{p} \\
0>v(c) & \geq-2 V_{m} \\
v(d) & \geq 0
\end{aligned}
$$

We see that Equation 24 implies that $v(c) \geq-2 V_{p}$, which we would have to use instead of $v(c) \geq-2 V_{m}$ if we did not assume $V_{p}>V_{m}$. We have the measure

$$
\left(1-q^{-1}\right) \sum_{v(c)=-2 V_{m}}^{-1} q^{-v(c)}\left(\left\lfloor\frac{V_{m}+V_{p}+v(c)}{2}\right\rfloor-\left\lceil\frac{V_{m}-V_{p}}{2}\right\rceil+1\right)
$$

Case $(1,0)$ : the integral $\mathcal{O}_{\gamma}: h=V_{m}-1, \ldots,-V_{m}$

The assumptions for $h$ are equivalent to $E_{4}, E_{5} \in \mathfrak{o}$ and $E_{4}^{\text {stc }}, E_{5}^{\text {stc }} \in \mathfrak{o}$. Hence, any solution that makes $E_{i}$ integral will make $E_{i}^{\mathrm{stc}}$ integral except when $E_{7}$ is integral but $E_{7}^{\mathrm{stc}}$ is not, which is equivalent to $V_{m}+v(d)-h \geq 0$ but $V_{m}+v(d)-h-1<0$. In other words, $v(d)=-V_{m}+h$. Since $h \leq V_{m}-1$ by assumption, this implies $v(d)<0$. Under this additional requirement, we reduce to the following.

$$
\begin{aligned}
2 h+V_{p}-V_{m} & \geq 2 v\left(r_{1}\right) \\
2 h+V_{p}-V_{m}+v(c) & \geq 2 v\left(r_{1}\right) \\
2 v\left(r_{1}\right) & \geq h-V_{p} \\
v(c) & \geq-h-V_{m} \\
v(d) & =h-V_{m}
\end{aligned}
$$

We see again that there are two cases: $v(c) \geq 0$ and $v(c)<0$.

Case 1. $v(c) \geq 0$. Then, the inequalities reduce to the product set defined by

$$
\begin{aligned}
2 h+V_{p}-V_{m} \geq 2 v\left(r_{1}\right) & \geq h-V_{p} \\
v(c) & \geq 0 \\
v(d) & =h-V_{m}
\end{aligned}
$$

Hence, the measure here is

$$
q^{V_{m}-h}\left(1-q^{-1}\right)\left(\left\lfloor\frac{2 h+V_{p}-V_{m}}{2}\right\rfloor-\left\lceil\frac{h-V_{p}}{2}+1\right\rceil\right)
$$

Case 2. $v(c)<0$. The relevant inequalities are

$$
\begin{aligned}
2 h+V_{p}-V_{m}+v(c) \geq 2 v\left(r_{1}\right) & \geq h-V_{p} \\
0>v(c) & \geq-h-V_{m} \\
v(d) & =h-V_{m}
\end{aligned}
$$


Hence, the measure of the corresponding set is

$$
q^{V_{m}-h}\left(1-q^{-1}\right)^{2} \sum_{v(c)=-h-V_{m}}^{-1} q^{-v(c)}\left(\left\lfloor\frac{2 h+V_{p}-V_{m}+v(c)}{2}\right\rfloor-\left\lceil\frac{h-V_{p}}{2}+1\right\rceil\right)
$$

Case $(0,1):$ the integral $\mathcal{O}_{\gamma_{\text {stc }}}: h=-V_{m}-1$

Since $h=-V_{m}-1$, the expressions $E_{*}$ cannot all be integral. Because $V_{m}+v(c)+h+1 \geq 0$, putting $h=-V_{m}-1$ into this gives $v(c) \geq 0$. We are left with

$$
\begin{aligned}
-V_{m}-1+V_{p}-2 v\left(r_{1}\right) & \geq 0 \\
-V_{m}-1+V_{p}+v(d)-2 v\left(r_{1}\right) & \geq 0 \\
V_{p}+V_{m}+1+2 v\left(r_{1}\right) & \geq 0 \\
v(c) & \geq 0 \\
v(d) & \geq-2 V_{m}
\end{aligned}
$$

We do two cases: $v(d) \geq 0$ and $v(d)<0$.

Case 1. $v(d) \geq 0$. We have the following:

$$
\begin{aligned}
V_{p}-V_{m}-1 & \geq 2 v\left(r_{1}\right) \geq-V_{p}-V_{m}-1 \\
v(c) & \geq 0 \\
v(d) & \geq 0
\end{aligned}
$$

The measure of this set is

$$
\begin{cases}V_{p} & \text { if } V_{m}+V_{p} \text { is even } \\ V_{p}+1 & \text { if } V_{m}+V_{p} \text { is odd }\end{cases}
$$

Case 2. $v(d)<0$.

$$
\begin{aligned}
& V_{p}-V_{m}-1+v(d) \geq 2 v\left(r_{1}\right) \geq-V_{p}-V_{m}-1 \\
& v(c) \geq 0 \\
& 0>v(d) \geq-2 V_{m}
\end{aligned}
$$

Transitivity in Equation 27 shows that $v(d) \geq-2 V_{p}$, but this is already satisfied under our hypothesis $V_{p}>V_{m}$. We see that the measure of this set is

$$
\left(1-q^{-1}\right) \sum_{v(d)=-2 V_{m}}^{-1} q^{-v(d)}\left(\left\lfloor\frac{V_{p}-V_{m}-1+v(d)}{2}\right\rfloor-\left\lceil\frac{-V_{p}-V_{m}-1}{2}+1\right\rceil\right)
$$

Case $(0,1):$ the integral $\mathcal{O}_{\gamma_{\mathrm{stc}}}: h=V_{m}-1, \ldots,-V_{m}$

We just need to evaluate under the conditions that each $E_{i}^{\text {stc }}$ is integral but at least one of $E_{i}$ is not. The only way this can happen is when $v(c)=-V_{m}-h-1$. In particular, this implies that $v(c)<0$. 
We start with the following (in)equalities:

$$
\begin{aligned}
h+V_{p}+v(c)-2 v\left(r_{1}\right) & \geq 0 \\
h+V_{p}+v(c)+v(d)-2 v\left(r_{1}\right) & \geq 0 \\
V_{p}-h+2 v\left(r_{1}\right) & \geq 0 \\
V_{m}+v(c)+h+1 & =0 \\
V_{m}+v(d)-h-1 & \geq 0
\end{aligned}
$$

We do two cases: $v(d) \geq 0$ and $v(d)<0$.

Case 1. $v(d) \geq 0$. Then, taking the above inequalities, eliminating the redundant ones (Equations 31 and 34) and putting $v(c)=-V_{m}-h-1$ gives

$$
\begin{aligned}
V_{p}-V_{m}-1 \geq 2 v\left(r_{1}\right) & \geq h-V_{p} \\
v(d) & \geq 0 \\
v(c) & =-V_{m}-h-1 .
\end{aligned}
$$

The measure of this set is then

$$
q^{V_{m}+h+1}\left(1-q^{-1}\right)\left(\left\lfloor\frac{V_{p}-V_{m}-1}{2}\right\rfloor-\left\lceil\frac{h-V_{p}}{2}+1\right\rceil\right)
$$

Case 2. $v(d)<0$. We again take Equation 30 to Equation 34, eliminate the redundant Equation 30 and make the substitution $v(c)=-V_{m}-h-1$ to get:

$$
\begin{aligned}
V_{p}-V_{m}-1+v(d) \geq 2 v\left(r_{1}\right) & \geq h-V_{p} \\
0>v(d) & \geq h+1-V_{m} \\
v(c) & =-V_{m}-h-1
\end{aligned}
$$

Giving us the measure

$$
q^{V_{m}+h+1}\left(1-q^{-1}\right)^{2} \sum_{v(d)=h+1-V_{m}}^{-1} q^{-v(d)}\left(\left\lfloor\frac{V_{p}-V_{m}-1+v(d)}{2}\right\rfloor-\left\lceil\frac{h-V_{p}}{2}+1\right\rceil\right)
$$

\section{All summands integral: taking the difference}

In this section, we take the measure we have found so far for case $(1,0)$ and subtract from it the measure for case $(0,1)$.

Extreme cases: $h=V_{m}$ for $\mathcal{O}_{\gamma}$ and $h=-V_{m}-1$ for $\mathcal{O}_{\gamma_{\text {stc }}}$

Here, we subtract from the measure for $h=V_{m}$ in $\mathcal{O}_{\gamma}$ the measure for $h=-V_{m}-1$ in $\mathcal{O}_{\gamma_{\text {stc }}}$, for all summands being integral. We find the difference to be:

$$
\begin{aligned}
(-1)^{V_{m}+V_{p}} & +\left(1-q^{-1}\right) \sum_{j=1}^{2 V_{m}} q^{j}\left(\left\lfloor\frac{V_{m}+V_{p}-j}{2}\right\rfloor-\left\lceil\frac{V_{m}-V_{p}}{2}\right\rceil-\left\lfloor\frac{V_{p}-V_{m}-1-j}{2}\right\rfloor\right. \\
& \left.+\left\lceil\frac{-V_{p}-V_{m}-1}{2}\right\rceil\right)
\end{aligned}
$$


In the summation, we see that the terms where $j$ is odd vanish, leaving us with:

$$
\begin{aligned}
(-1)^{V_{m}+V_{p}} & +\left(1-q^{-1}\right) \sum_{j=1}^{V_{m}} q^{2 j}\left(\left\lfloor\frac{V_{m}+V_{p}-2 j}{2}\right\rfloor-\left\lceil\frac{V_{m}-V_{p}}{2}\right\rceil\right. \\
& \left.-\left\lfloor\frac{V_{p}-V_{m}-1-2 j}{2}\right\rfloor+\left\lceil\frac{-V_{p}-V_{m}-1}{2}\right\rceil\right)
\end{aligned}
$$

Simplifying the floor and ceiling functions gives $(-1)^{V_{m}+V_{p}}$, so that we get

$$
(-1)^{V_{m}+V_{p}}\left(1+\left(1-q^{-1}\right) \sum_{j=1}^{V_{m}} q^{2 j}\right)=(-1)^{V_{m}+V_{p}}\left(1+\left(q^{2 V_{m}}-1\right) \frac{q}{q+1}\right) \text {. }
$$

$h=V_{m}-1, \ldots,-V_{m}$

There were two cases here: the first, where $v(c) \geq 0$ for $\mathcal{O}_{\gamma}$ and $v(d) \geq 0$ for $\mathcal{O}_{\gamma_{\text {stc }}}$ and the second (reverse the inequalities).

Case 1. When $v(c) \geq 0$ for $\mathcal{O}_{\gamma}$ and $v(d) \geq 0$ for $\mathcal{O}_{\gamma_{\text {stc }}}$. In this case, we had for $\mathcal{O}_{\gamma}$ the measure:

$$
q^{V_{m^{-h}}}\left(1-q^{-1}\right)\left(\left\lfloor\frac{2 h+V_{p}-V_{m}}{2}\right\rfloor-\left\lceil\frac{h-V_{p}}{2}\right\rceil+1\right)
$$

and for $\mathcal{O}_{\gamma_{\text {stc }}}$ :

$$
q^{V^{+h+1}\left(1-q^{-1}\right)}\left(\left\lfloor\frac{V_{p}-V_{m^{-1}}}{2}\right\rfloor-\left\lceil\frac{h-V_{p}}{2}\right\rceil+1\right)
$$

We have to sum both over $h=-V_{m}, \ldots, V_{m}-1$ and subtract the second from the first. However, in placing this in the summation, we may replace $h$ in the second expression with $-h-1$, a transformation which preserves the summation range. We do this since then we will get pairs of nicely paired terms in the sum. So we get:

$$
\begin{aligned}
& q^{V_{m}}\left(1-q^{-1}\right) \sum_{h=-V_{m}}^{V_{m}-1} q^{-h}\left(\left\lfloor\frac{2 h+V_{p}-V_{m}}{2}\right\rfloor-\left\lceil\frac{V_{p}-V_{m}-2}{2}\right\rceil\right. \\
&\left.+\left\lfloor\frac{-h-V_{p}}{2}\right\rfloor-\left\lceil\frac{h-V_{p}}{2}\right\rceil\right)
\end{aligned}
$$

where we have converted the floor to ceiling and vice version in the second equation to make following the computations with 'Integer arithmetic' easier. We break the summation into two sums: one over $h=-V_{m},-V_{m}-2, \ldots, V_{m}-2$ and the other over $h=-V_{m}+1, \ldots, V_{m}-1$. In other words, the first is over integers of the same parity as $V_{m}$ and the second opposite parity. However, if we look at the opposite-parity case, we see that $2 h+V_{p}-V_{m}$ and $V_{p}-V_{m}-2$ have the same parity, which is the opposite parity of both $-h-V_{p}$ and $h-V_{p}$. Hence, the sum vanishes. So, we just have sum over $h=-V_{m},-V_{m}+2, \ldots, V_{m}-2$. In this case, we get

$$
\begin{aligned}
& (-1)^{V_{m}+V_{p}} q^{V_{m}}\left(1-q^{-1}\right)\left(q^{V_{m}}+q^{V_{m}-2}+\cdots+q^{-V_{m}+2}\right) \\
= & (-1)^{V_{m}+V_{p}} q^{2}\left(1-q^{-1}\right)\left(1+q^{2}+\cdots+\left(q^{2}\right)^{V_{m}-1}\right) \\
= & (-1)^{V_{m}+V_{p}}\left(q^{2 V_{m}}-1\right) \frac{q}{q+1}
\end{aligned}
$$


Case 2. When $v(c)<0$ for $\mathcal{O}_{\gamma}$ and $v(d)<0$ for $\mathcal{O}_{\gamma_{\text {stc }}}$. This is a little more lengthy but not terribly so. We recall the two terms. The first for $\mathcal{O}_{\gamma}$ is

$$
q^{V_{m}-h}\left(1-q^{-1}\right)^{2} \sum_{v(c)=-h-V_{m}}^{-1} q^{-v(c)}\left(\left\lfloor\frac{2 h+V_{p}-V_{m}+v(c)}{2}\right\rfloor-\left\lceil\frac{h-V_{p}}{2}\right\rceil+1\right)
$$

The one for $\mathcal{O}_{\gamma_{\text {stc }}}$ is

$$
q^{V_{m}+h+1}\left(1-q^{-1}\right)^{2} \sum_{v(d)=h+1-V_{m}}^{-1} q^{-v(d)}\left(\left\lfloor\frac{V_{p}-V_{m}-1+v(d)}{2}\right\rfloor-\left\lceil\frac{h-V_{p}}{2}\right\rceil+1\right)
$$

The first thing we do is replace $h$ by $-h-1$ in the $\gamma_{\text {stc }}$ version and use $j$ as the index of summation over positive instead of negative numbers. After doing this, converting the appropriate floors to ceilings and vice versa and subtracting the second from the first, we get:

$q^{V_{m}-h}\left(1-q^{-1}\right)^{2} \sum_{j=1}^{V_{m}+h} q^{j}\left(\left\lfloor\frac{2 h+V_{p}-V_{m}-j}{2}\right\rfloor-\left\lceil\frac{V_{p}-V_{m}-2-j}{2}\right\rceil+\left\lfloor\frac{-h-V_{p}}{2}\right\rfloor-\left\lceil\frac{h-V_{p}}{2}\right\rceil\right)$

Of course, we have still not summed over $h$ yet; and we also note that if $h=-V_{m}$, the sum is actually empty. Anyways, to make sense of this chaos, we write down two separate summations again: one for $h=-V_{m},-V_{m}+2, \ldots, V_{m}-2$ and one for $h=$ $-V_{m}+1,-V_{m}+3, \ldots, V_{m}-1$.

Case 2.1. $h=-V_{m},-V_{m}+2, \ldots, V_{m}-2$. Here, the upper limit of the summation is even. We also split the summation into two sums, depending on whether $j$ is even or odd:

$$
\begin{aligned}
q^{V_{m}-h}\left(1-q^{-1}\right)^{2} & {\left[\sum _ { j = 1 } ^ { \frac { V _ { m } + h } { 2 } } q ^ { 2 j } \left(\left\lfloor\frac{2 h+V_{p}-V_{m}-2 j}{2}\right\rfloor-\left\lceil\frac{V_{p}-V_{m}-2-2 j}{2}\right\rceil\right.\right.} \\
& \left.+\left\lfloor\frac{-h-V_{p}}{2}\right\rfloor-\left\lceil\frac{h-V_{p}}{2}\right\rceil\right)+\sum_{j=1}^{\frac{V_{m}+h}{2}} q^{2 j-1}\left(\left\lfloor\frac{2 h+V_{p}-V_{m}-2 j+1}{2}\right\rfloor\right. \\
& \left.\left.-\left\lceil\frac{V_{p}-V_{m}-1-2 j}{2}\right\rceil+\left\lfloor\frac{-h-V_{p}}{2}\right\rfloor-\left\lceil\frac{h-V_{p}}{2}\right\rceil\right)\right]
\end{aligned}
$$

We again see that the summation where $j$ is odd vanishes, and we simplify the rest to get

$$
\begin{aligned}
(-1)^{V_{m}+V_{p}} q^{V_{m}-h}\left(1-q^{-1}\right)^{2} \sum_{j=1}^{\frac{V_{m}+h}{2}} q^{2 j} & =(-1)^{V_{m}+V_{p}} q^{V_{m}-h}\left(1-q^{-1}\right)^{2} q^{2} \frac{q^{V_{m}+h}-1}{q^{2}-1} \\
& =(-1)^{V_{m}+V_{p}} \frac{q-1}{q+1}\left(q^{2 V_{m}}-q^{V_{m}-h}\right)
\end{aligned}
$$

As a sanity check, putting in $h=-V_{m}$ gives zero. Good! Let us sum over $h$ now to get

$$
\begin{aligned}
& (-1)^{V_{m}+V_{p}}\left[\frac{q-1}{q+1} q^{2 V_{m}}\left(V_{m}\right)-\frac{q-1}{q+1} q^{V_{m}}\left(q^{V_{m}}+q^{V_{m}-2}+q^{V_{m}-4}+\cdots+q^{-V_{m}+2}\right)\right] \\
= & (-1)^{V_{m}+V_{p}}\left[\frac{q-1}{q+1} q^{2 V_{m}}\left(V_{m}\right)-\frac{q^{2}}{(q+1)^{2}}\left(q^{2 V_{m}}-1\right)\right]
\end{aligned}
$$


Case 2.2. $h=-V_{m}+1,-V_{m}+3 \cdots, V_{m}-1$. This time, the upper limit $V_{m}+h$ is odd. We again split the summation into two sums, depending on whether $j$ is odd or even:

$$
\begin{aligned}
q^{V_{m}-h}\left(1-q^{-1}\right)^{2}\left[\sum _ { j = 1 } ^ { \frac { V _ { m } + h - 1 } { 2 } } q ^ { 2 j } \left(\left\lfloor\frac{2 h+V_{p}-V_{m}-2 j}{2}\right\rfloor-\left\lceil\frac{V_{p}-V_{m}-2-2 j}{2}\right\rceil\right.\right. \\
\left.+\left\lfloor\frac{-h-V_{p}}{2}\right\rfloor-\left\lceil\frac{h-V_{p}}{2}\right\rceil\right)+\sum_{j=1}^{\frac{V_{m}+h+1}{2}} q^{2 j-1}\left(\left\lfloor\frac{2 h+V_{p}-V_{m}-2 j+1}{2}\right\rfloor\right. \\
\left.\left.-\left\lceil\frac{V_{p}-V_{m}-1-2 j}{2}\right\rceil+\left\lfloor\frac{-h-V_{p}}{2}\right\rfloor-\left\lceil\frac{h-V_{p}}{2}\right\rceil\right)\right]
\end{aligned}
$$

This time, the opposite happens: in other words, the summation with even powers of $q$ vanishes, and we are left with

$$
-(-1)^{V_{m}+V_{p}} q^{V_{m}-h}\left(1-q^{-1}\right)^{2} \sum_{j=1}^{\frac{V_{m}+h+1}{2}} q^{2 j-1}=-(-1)^{V_{m}+V_{p}} \frac{q-1}{q+1}\left(q^{2 V_{m}}-q^{V_{m}-h-1}\right)
$$

Summing over $h=-V_{m}+1,-V_{m}+3, \cdots, V_{m}-1$ gives

$$
-(-1)^{V_{m}+V_{p}}\left[\frac{q-1}{q+1} q^{2 V_{m}}\left(V_{m}\right)-\frac{1}{(q+1)^{2}}\left(q^{2 V_{m}}-1\right)\right]
$$

We add this to Equation 35 to get

$$
(-1)^{V_{m}+V_{p}}\left[\frac{1}{(q+1)^{2}}\left(q^{2 V_{m}}-1\right)-\frac{q^{2}}{(q+1)^{2}}\left(q^{2 V_{m}}-1\right)\right]=\left(q^{2 V_{m}}-1\right) \frac{1-q}{1+q}
$$

\section{Gathering of terms}

We have now collected all the terms in our integral for the 'all summands positive' case.

We add them together:

$$
\begin{aligned}
&(-1)^{V_{m}+V_{p}} {\left[1+\left(q^{2 V_{m}}-1\right) \frac{q}{q+1}+\left(q^{2 V_{m}}-1\right) \frac{q}{q+1}+\left(q^{2 V_{m}}-1\right) \frac{1-q}{q+1}\right] } \\
&=(-1)^{V_{m}+V_{p}} q^{2 V_{m}} .
\end{aligned}
$$

\section{Summands of $E_{6}$ integral only}

The last case is the case of the summands of $E_{6}$ being integral only. This case is a little different because here, it will be impossible that both $E_{7}$ and $E_{7}^{\text {stc }}$ will be simultaneously satisfied. 
Lemma 4.6.1. For the summands of $E_{6}=E_{6}^{\text {stc }}$ to be integral and the summands of $E_{7}$ (resp. $\left.E_{7}^{\text {stc }}\right)$ to be not integral, the following inequalities have to be satisfied:

$$
\begin{aligned}
& \text { For } \mathcal{O}_{\gamma} \quad \text { (resp. for } \mathcal{O}_{\gamma_{\text {stc }}} \text { ) } \\
& h+V_{p}-2 v\left(r_{1}\right) \geq 0 \\
& h+V_{p}+v(c)-2 v\left(r_{1}\right) \geq 0 \\
& h+V_{p}+v(d)-2 v\left(r_{1}\right) \geq 0 \\
& h+V_{p}+v(c)+v(d)-2 v\left(r_{1}\right) \geq 0 \\
& V_{p}-h+2 v\left(r_{1}\right) \geq 0 \\
& V_{m}+v(c)+h<0 \\
& V_{m}+v(d)-h<0 \\
& \begin{aligned}
V_{m}+v(c)+h+1 & <0 \\
V_{m}+v(d)-h-1 & <0
\end{aligned}
\end{aligned}
$$

Proof. We set the valuations of the expressions at the beginning of 'Elimination of fiendish cases' to be greater than or equal to zero for $E_{1}$ to $E_{5}$, and we do the same for the summands of $E_{6}$. We also set the summands of $E_{7}$ (resp. $E_{7}^{\text {stc }}$ ) to have valuation less than zero. As usual, we have omitted the inequalities for $E_{4}, E_{4}^{\mathrm{stc}}, E_{5}, E_{5}^{\mathrm{stc}}$ since these are equivalent to our assumptions on $h$.

\section{Case $(1,0)$ : the integral $\mathcal{O}_{\gamma}$}

Here, we come up with an expression for $h=-V_{m}, \ldots, V_{m}$. We make three straightforward observations:

1. Since $V_{m}+h+v(c)<0$ and $V_{m}+h \geq 0$, we must have $v(c)<0$, and similarly, $V_{m}-h+v(d)<0$ implies that $v(d)<0$

2. Since the summands of $E_{7}$ are not integral, we must have the valuations of these terms equal. Hence, $v(d)-2 h=v(c)$.

3. In addition, since we are solving a congruence in $E_{7}$, once $v(d)$ is chosen, the measure of $\left\{c: v\left(E_{7}\right) \geq 0\right\}$ is $q^{V_{m}+h}$.

Making the substitution $v(c)=v(d)-2 h$ and eliminating redundancies gives the inequalities

$$
\begin{aligned}
V_{p}-h+2 v(d) \geq 2 v\left(r_{1}\right) & \geq h-V_{p} \\
h-V_{m} & >v(d)
\end{aligned}
$$

We see from the first that $v(d) \geq h-V_{p}$, which gives a lower limit for $v(d)$. Thus, the measure of this solution set is

$$
q^{V_{m}+h}\left(1-q^{-1}\right) \sum_{v(d)=h-V_{p}}^{h-V_{m}-1} q^{-v(d)}\left(\left\lfloor\frac{V_{p}-h+2 v(d)}{2}\right\rfloor-\left\lceil\frac{h-V_{p}}{2}+1\right\rceil\right) .
$$

\section{Case $(0,1)$ : the integral $\mathcal{O}_{\gamma_{\text {stc }}}$}

Here, we have essentially the same three observations as in 'Case $(1,0)$ : the integral $\mathcal{O}_{\gamma}$ ', suitably modified.

1. Since $V_{m}+h+v(c)+1<0$ and $V_{m}+h+1 \geq 0$, we must have $v(c)<0$, and similarly, $V_{m}-h+v(d)-1<0$ implies that $v(d)<0$ 
2. Since the summands of $E_{7}^{\text {stc }}$ are not integral, we must have the valuations of these terms equal. Hence, $v(d)-2 h-2=v(c)$.

3. In addition, since we are solving a congruence in $E_{7}^{\text {stc }}$, once $v(d)$ is chosen, the measure of $\left\{c: v\left(E_{7}\right) \geq 0\right\}$ is $q^{V_{m}+h+1}$.

We have the inequalities:

$$
\begin{aligned}
V_{p}-h+2 v(d)-2 \geq 2 v\left(r_{1}\right) & \geq h-V_{p} \\
h+1-V_{m}>v(d) & \geq h-V_{p}+1
\end{aligned}
$$

where the lower limit for $v(d)$ comes from the first inequality. We obtain the measure

$$
q^{V_{m}+h+1}\left(1-q^{-1}\right) \sum_{v(d)=h-V_{p}+1}^{h-V_{m}} q^{-v(d)}\left(\left\lfloor\frac{V_{p}-h+2 v(d)-2}{2}\right\rfloor-\left\lceil\frac{h-V_{p}}{2}+1\right\rceil\right)
$$

\section{Gathering it together}

In this case, we see that after shifting the index of summation in Equation 37 so that $v(d)$ starts at $h-V_{p}$, we get exactly Equation 36 , so that the two cancel.

\section{Results and interpretations}

The calculations of 'Brute force calculations' section, shown particularly in 'Gathering of terms', give

$$
\mathcal{O}_{\gamma}^{\kappa}(\mathbf{1})=(-1)^{V_{m}+V_{p}} q^{2 V_{m}}
$$

We recall that $\gamma=\operatorname{diag}(x, y,-y,-x)$ and $V_{m}=v(x-y)$. We have suggested that the corresponding endoscopic space $\left(H, \theta_{H}\right)$ is two copies of $\mathrm{U}(1) \times \mathrm{U}(1) \hookrightarrow \mathrm{U}(2)$, given as follows. Set $J_{2}=\left(\begin{array}{ll}0 & 1 \\ 1 & 0\end{array}\right)$. We have $\mathrm{U}(2):=\left\{g \in \mathrm{GL}_{2}: J \bar{g}^{-t} J=g\right\}$ and $\mathrm{U}_{2}^{J_{2}} \cong \mathrm{U}(1) \times \mathrm{U}(1)$, so that $\theta_{H}=\left(J_{2}, J_{2}\right)$. A trivial computation shows that for $\gamma_{H}=(\operatorname{diag}(x,-x), \operatorname{diag}(y,-y))$, the corresponding stable orbital integral is just one. Although this does not suggest a way to define endoscopic pairs $\left(H, \theta_{H}\right)$ in general, it is the likely choice given the situation with the adjoint case. Hence:

Theorem 5.0.5. For $\left(\mathrm{U}_{4}, \theta\right)$ and $\left(\mathrm{U}_{2} \times \mathrm{U}_{2}, \theta_{H}\right)$ with $\gamma=\operatorname{diag}(x, y,-y,-x)$ nearly singular and $\gamma_{H}=\operatorname{diag}(x,-x) \times \operatorname{diag}(y,-y)$, we have the identity

$$
\mathcal{O}_{\gamma}^{\kappa}\left(\mathbf{1}_{\mathfrak{g}_{1}(\mathfrak{o})}\right)=(-1)^{V_{m}+V_{p}} q^{2 V_{m}} \mathcal{S} \mathcal{O}_{\gamma_{H}}\left(\mathbf{1}_{\mathfrak{h}_{1}(\mathfrak{o})}\right)
$$

The factor of $(-1)^{V_{m}+V_{p}}$ is not terribly mysterious, and one could likely eliminate it by using the relative Kostant-Weierstrass section shown to exist in [4] and following the ideas in [3], so we concentrate on the power of $q$. We offer the following tentative interpretation of the power of $q$.

We define $\tilde{\gamma} \in \mathfrak{u}^{\theta}(F)$ by $\lambda \operatorname{diag}(x, y, y, x)$ where $\lambda \in E$ is such that $v(\lambda)=0$ and $\bar{\lambda}=-\lambda$. The map $\gamma \mapsto \tilde{\gamma}$ gives an $F$-linear isomorphism between $\operatorname{Lie}\left(I_{\gamma}\right)(F)$ and $\mathfrak{a}(F)$. But centraliser $I_{\gamma}$ of $\gamma$ in $G_{0}$ is $I_{\gamma}=\left\{\operatorname{diag}\left(a_{11}, a_{22}, a_{22}, a_{11}\right): a_{i i} \bar{a}_{i i}=1\right\}$. Its roots, or 
nonzero weights, of its action on $\mathfrak{g}_{1}$ (and on $\mathfrak{g}_{0}$ ) are given (in terms of homomorphisms to $\operatorname{Res}_{E / F}\left(\mathbb{G}_{m}\right)$, using adjointness of the restriction of scalars) by

$$
\begin{aligned}
& \operatorname{diag}\left(a_{11}, a_{22}, a_{22}, a_{11}\right) \mapsto a_{11} a_{22}^{-1}, \\
& \operatorname{diag}\left(a_{11}, a_{22}, a_{22}, a_{11}\right) \mapsto a_{11}^{-1} a_{22} .
\end{aligned}
$$

Each root space being two dimensional. Let $D: \operatorname{Lie}\left(I_{\gamma}\right) \rightarrow F \delta$ be the discriminant function $\prod_{\alpha}(d \alpha)^{r_{\alpha}}$ where $r_{\alpha}$ is the $F$ dimension of the corresponding root space. Naïvely using the formula in [5] with this discriminant function on $\tilde{\gamma}$ gives

$$
\begin{aligned}
D(\tilde{\gamma}) / 2 & =\frac{2 v(x-y)+2 v(x-y)}{2} \\
& =2 V_{m} .
\end{aligned}
$$

We shall attempt a reasonable explanation in a future work.

\section{Competing interests}

The author declares that he has no competing interests.

\section{Acknowledgements}

The author would like to thank Jayce R. Getz for suggesting this problem, for a thorough reading of the manuscript and for encouragement. The author also thanks the anonymous referee for suggesting some improvements to the exposition and pointing out a typographical error.

Received: 22 December 2014 Accepted: 8 March 2015

Published online: 10 July 2015

\section{References}

1. Borovoi M: Abelian Galois Cohomology of Reductive Groups vol. 626. American Mathematical Society, 201 Charles Street, Providence, RI. USA 02904-2294 (1998)

2. Getz, JR, Wambach, E: Twisted relative trace formulae with a view towards unitary groups. Amer. J. Math. 135, 1-57 (2013)

3. Kottwitz, R: Transfer factors for lie algebras. Representation Theory Am. Math. Soc. 3(6), 127-138 (1999)

4. Levy, P: Involutions of reductive lie algebras in positive characteristic. Adv. Mathematics. 210(2), 505-559 (2007)

5. Ngô, BC: Le lemme fondamental pour les algebres de lie. Publications mathématiques de l'IHÉS. 111(1), 1-169 (2010)

\section{Submit your manuscript to a SpringerOpen ${ }^{\odot}$ journal and benefit from:}

$\rightarrow$ Convenient online submission

$\rightarrow$ Rigorous peer review

- Immediate publication on acceptance

- Open access: articles freely available online

- High visibility within the field

- Retaining the copyright to your article 